Sohreiben wir

$$
p^{\prime}=-\left(T_{x \frac{\partial^{2} z}{\partial x^{2}}}+T_{y} \frac{\partial^{2} z}{\partial y^{2}}+2 T_{x y} \frac{\partial^{2} z}{\partial x \partial y}\right)
$$

so erhalten wir

$$
\frac{m^{2} E J}{m^{2}-1}\left\{\frac{\partial^{4} z}{\partial x^{4}}+2 \frac{\partial^{4} z}{\partial x^{7} \partial y^{2}}+\frac{i)^{4} z}{\partial y^{4}}\right\}=p-p^{\prime} \text {. }
$$

$p^{\prime}$ können wir als den Teil der Belastung denken, welcher von den in der Plaite vorhandenen Zag- und Schabspannungsresultanten aufgenommen wird, ohne daB die Biegungsfestigkeit dabei ins Spiel käme.

Eine Berechnung derartiger Platten ist nur möglich, wenn das Verhältnis $\left(\frac{a}{b}\right)^{4}:\left(\frac{E}{p}\right)$ bekannt ist, so dab man daran denken könnte, Kurventafeln für verschiedene Werte dieses Verhultnisses zu berechnen. Hier wollen wir uns jedoch nur mit der Frage beschaftigen, inwieweit unsere Annahme einer Platte ohne Biegungssteifigkeit berechHolienschichtenkarte der verzerrten Mittelfluche der Platte b) $\operatorname{nach}$ Gl, 1)

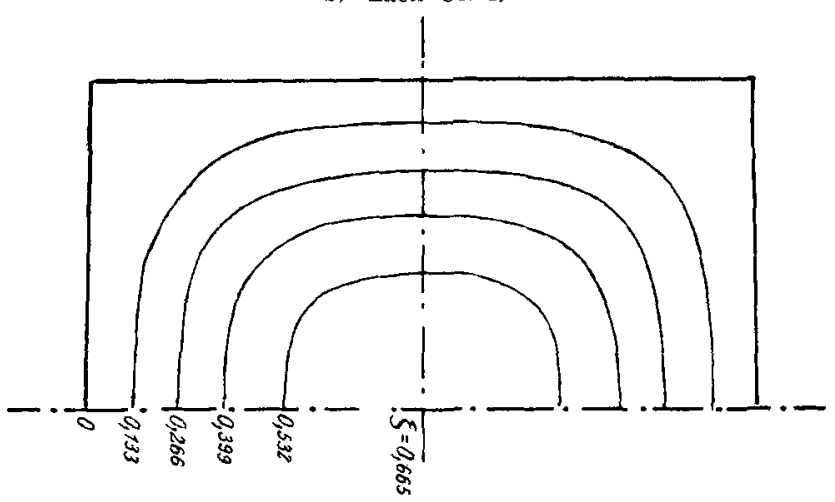

Abb. 5 b $T_{x y}=0$ und die Ausdrücke $T_{2} \frac{\partial^{2} z}{\partial x_{2}}$ sowie $T_{y} \frac{\partial^{2} z}{\partial y^{2}}$ verschwinden ebenfalls; wie klein auch das Trägheitsmoment der Platte sein mag, so tritt doch eine Fntlastung der biegungssteifen Platte infolge der Verzerrung der Mittelfläche unmittelbar am Rand nicht ein. Bei der eingespannten und gleichzeitig festgehaltenen Platte tritt auch an den Randern eine Entlastung ein, welche aber an den Ecken der Platte verschwindet. Es ergibt sich darans, daß unsere Berechnung nur mit einer gewissen Einschränkung aul wirklich vorkommende Platten übertragen werden darf.

Der Mangel einer einwandireien Lösung des Plattenproblems hat besonders bei der Beurteilung der Versuchsergebnlsse sehr nachteilig gewirkt. Die Entlastung der Platte von Biegungsarbeit ist nämlich schon bei verhăltnismäßig steifen Platten ein nicht za vernachlassigender Umstand. Sogar bei einer freigelagerten Platte bildet sich an den Rändern ein Druckring aus, in welchen sich der mittlere Teil der Platte gewissermaßen einhängt.

$\mathrm{Zu}$ beachten ist auch, daß sich die Fülle von Einspannungs- und Auflagerrungsmöglichkeiten allein durch Einführung erfahrungsmäßiger Berichtigangskoeffizienten wohl nicht bewältigen läBt.

Es wäre sebr zu begrüßen, wenn auch für mühsame, aber praktisch wichtige rechnerische Arbeiten auf dem Gebiet der Festigkeitslehre Mittel bereitgestellt würden, wie es für Versuchszwecke längst geschieht, denn beide Arbeitsrichtangen können nur durch ihr Zusammenwirken wertvolle Ergebnisse zustande bringen

\title{
Über die Berechnung der Schubspannungen im gebogenen Balken ${ }^{1}$.
}

Von P. NEMÉNYI in Budapest.

$\mathrm{D}$ ie von Saint-Venant begründete Theorie der Torsion prismatischer Stabe hat zu zahlreichen Methoden geführt, die für die Ermittlung der Spannungsverteilung in einem auf Torsion beanspruchten Stabe von beliebigem Querschnitt geeignet sind, oder die es mindestens ormöglichen, uns über diese eine zutrefiende Vorstellung zu

1) Vorllegende Abhandlunk ist eine, dem Wesen nach unveränderte deutsche Bearbeltung jneineg ungarischen Aufeatzes, welcher am 15. März I, J, der Sitzung der III. Klasse der Ungarischen Akademie der Wissenschaften vorgelegt wurde. 
bilden. Die bydrodynamischen Analogien "), die Prand tlsche Analogie mit der gespannten Membran ${ }^{2}$ ), die Hennebergschen Untersuchungen über die Verteilung der sogenannten Spannungsmassen ${ }^{3}$ ) und schlisblich Runges, auf Grundlage der Prandtlschen Darstellungsweise avfgebautes, numerisch-graphisches Verfahren ${ }^{4}$ ) sind alle danach angetan, um - auf dem festen Boden der Saint-Venantschen Lösung - eine richtige, entweder approximativ-numerische oder experimentell-anschauliche Lösung des Torsionsproblems zu bieten, auch für solche Stabquerschnitte, für welche die Lösung weder in geschlossener analytischer Form, noch in Form von Reihenentwicklungen gegeben werden kann.

Hingegen entbehrt die ebenfalls durch Saint-Venant begründete Theorie der Schubspaningen im Querschnitt des durch eine Einzellast gebogenen Stabes fast vollstandig derartiger Methoden. Das in der Ingenieurpraxis vielfach bonutzte, auf der willkïrlichen Annabme der Affinität zwischen Querschnittsbegrenzung und Schubspannungstrajektorien beruhende, angebliche *Näherungsverfahren " verdient diese Bezeichnung durchaus nicht; nicht nur das Mafs der etwaigen Annäherung bleibt uns bei diesem Verfahren vollständig unbekannt, sondern es führt schon in den allereinfachsten Fällen, z. B. beim Rechteck, auf Ergebnisse, die der richtigen Lösung sogar qualitatio widersprechen ${ }^{5}$ ). Der Zweck der vorliegenden Abhandlung ist, diese Lücke der Elastizitätslehre auszufüllen, d. b. eine Methode zu entwickeln, die, an den Ergebnissen der Saint-Venantschen Theorie festhaltend, Näherungslösungen für die Verteilung der Schubspannungen im gebogenen Balken bei - zur Lastrichtung symmetrischen, sonst aber ganz beliebigen - eventuell zeichnerisch gegebenen Querschnitten ermöglicht.

Die Schwierigkeit, die in der Biegnngstheorie gegenüber der Torsionstheorie besteht, hat darin ihren Grund, daß man zwar in beiden Fällen die Ermittlung der Spannungsverteilung auf die Aufsuchung einer; einer einfachen Differentialgleichung genügenden, Spannungsfunktion zurückführen kann, daß aber die Randbedingnng, der diese Funktion genügen muf, im Falle der Biegung sehr unübersichtlich und verwickelt erscheint. Es gelingt nun im folgenden (1), eine allgemeinere Spannungsfunktion $F$ für das Biegungsproblem zu definieren, die noch von zwei willkürlichen Größen $q_{1}$ und $p_{2}$ abhängt. Wie immer über diese Größen verfügt wird, auf jeden Fall wird die Differentialgleichung nur wenig verändert, wăhrend die Randbedingung die denkbar einfachste Form annimmt, nämlich in der unmittelbaren Vorgabe der Randwerte der gesuchten Funktion besteht; die Randwerte sind hierbei durch eine einfache Quadratur aus der Gestalt des Querschnittes abzuleiten. In 2 wird eine erste Annahme für $\varphi_{1}$ und $q_{3}$ erörtert, in 3 eine zweite, bei der die Differentialgleichung für $F$ die Laplacesche wird, so daß man analog dem Prandtlschen Gleichnis in der Torsionstheorie - eine experimentelle Lösnng des Biegungsproblems durch Herstellung eines gespannten Flüssigkeitshäutchens gewinnen kann. Natïrlich ist in allen Fällen das numerisch-graphisehe Verfabren, das R n ng $\theta$ zur Lösung des Torsionsproblems gewählt hat, auf die hier eingeführte Spannungsfunktion anwendbar. In 4 werden die Folgerungen aus dem neuen Ansatz für den Fall der * Grashofschen Querschnitte* gezogen und die Richtlinien der weiter in Angriff zu nehmenden Arbeiten angedeutet.

1. Einführung einer allgemeinen Spannungs-Funktion F. Ich wähle als Ausgangspunkt folgendes Ergebnis der Saint-Venantschen Biegungstheorie: Nehmen wir die Symmetrieachse des Querschnittes - welche mit der Lastrichtung zusammenfällt - zur $y$-Achse und die dazu senkrechte Schwerpunktsachse zar $x$.Achse, so lassen sich die Sohubspannungskomponenten folgendermaßen durch eine Spannungsfunktion $\Psi$ ansdrïcken ${ }^{6}$ ):

1) Love-Timpe, Lehrbuch d. Elastizitat, Leipzig 1907, S. 364.

3) Jahresb. d. deutschen Math. Vereinigung. Bd. 13, 1904. S. 31

3) Zeitschr. f. Math. u. Phys1k, Bd. 51, 1904, s. 242.

4) Zeltschr. F. Math. u. Physik, Bd. 56, 1908, s 225.

5) Love-Timpe, S 309. Love beweigt im AnschluB an die Grashofschen Untersuchungen, daß der durch diese Methode erhaltenen Spannungsverteilung $n \mathrm{n}$ bei einer bestimmten dreidimensionalen Schar von Querschnittsbegrenzungen ein geometriseh moglicher Deformationszustand entspricht.

6) Hans Lorenz, Lehrbuch d. techn. Pliysik. Bd IV, Müchen 1913, S. 440. Die Bezeich. nung der Spannungskmponenten ist aber die Fopplseha, mit zwel Indines. 


$$
\begin{aligned}
& \tau_{y z}=C\left(\frac{\partial \Psi}{\partial y}-\frac{y^{2}}{2}-\frac{2 m-1}{2} x^{2}\right) \\
& \tau_{x s}=C\left(\frac{a \Psi}{i x}-(2 m+1) x y\right)
\end{aligned}
$$

Hierin ist $C=\frac{1}{y m+1} \frac{P}{J_{x}}, P$ die Last, $J_{x}=\iint_{(P)} y^{2} d x d y$, das Trägheitsmoment in bezug auf die $x$-Achse und $m$ der Poissonsche Modul. Die Funktion $\Psi^{5}$ wird durch die partielle Differentialgleichung:

$$
L_{x y}{ }^{2} \Psi \equiv \frac{\partial^{2} \Psi}{\partial x^{2}}+\frac{i y^{2} y^{\prime}}{i y^{2}}=0
$$
und durch die Randbedingung

$$
\frac{\tau_{y z}}{\tau_{x z}}=\frac{\frac{\partial \Psi}{\partial y}-\frac{y^{2}}{2}-\frac{2 m-1}{2} x^{2}}{\frac{\partial \Psi}{\partial x}-(2 m+1) x y}=\frac{d y}{d x}
$$

(die besagt, daß die Randspannung die Richtung des Randes hat) definiert. Letztere läßt sich auch wie folgt schreiben, wenn man den Differentialquotienten $\frac{1 \Psi}{\partial_{n}}$ nach der Normalen des Randes

$$
\frac{\partial \Psi}{i_{n}} d s=\frac{\partial \Psi}{\partial y} d x-\frac{\partial \Psi}{\partial x} d y
$$

und den Winkel $\Phi$ zwischen Randtangente und $x$-Achse einführt:

$$
\frac{\partial}{i_{n}}=\cos \varphi\left(\frac{y^{2}}{2}+\frac{2 m-1}{2} x^{2}\right)-\sin \varphi(2 m+1) x y . . . \quad . \quad(d) \text {. }
$$

Diese Randbedingung läßt aber keine unmittelbare Folgerung in bezug auf die Randwerte der Funktion $\Psi$ za, und an diesem Umstande muBte bisher jeder Versuch scheitern, die Theorie in der Richtung der numerisch-graphischen oder sonstigen Approximationslösungen weiter auszubilden.

Um uns von dieser nachteiligen Randeigenschaft der Funktion $\Psi$ zu befreien und um gleichzeitig auch einen tieferen Einblick in das Wesen des Problems zu gewinnez, ersetze ich das Gleichungssystem ( $\psi / S$ ) durch folgenden, allgemeineren Ansatz:

$$
\left.\begin{array}{rl}
\tau_{y z} & =A_{1} \frac{\partial F}{\partial x}+B_{1} \frac{\partial F^{\prime}}{\partial y}+\varphi_{1}(x, y) \\
\tau_{x z} & =A_{2} \frac{\partial F}{\partial x}+B_{2} \frac{\partial F}{\partial y}+\varphi_{2}(x, y)
\end{array}\right\}
$$

wo $\varphi_{1}$ und $\Phi_{2}$ vorläufig unbekannte, von der Querschnittsiorm aber nicht abhängende Funktionen, $A_{1}, A_{2}, B_{1}, B_{2}$ aber ebensolche Konstanten sein sollen; und man siebt sofort, daB die spezielle Substitution $A_{1}=B_{2}=0, \quad A_{2}=B_{1}=C ; \quad q_{1}=-C\left(\frac{y^{2}}{2}+\frac{2 m-1}{2} x^{2}\right)$ und $\Phi_{2}=-C(2 m+1) x y$ das Gleichungssystem $(F)$ in ( $\left.\Psi^{\prime}\right)$ überführt.

Für den allgemeinen Ansatz (F) wird die Grenzbedingung zu:

$$
\frac{\tau_{y z}}{\tau_{x z}} \equiv \frac{A_{1} \frac{\partial F}{\partial x}+B_{1} \frac{\partial F}{\partial y}+\varphi_{1}(x, y)}{\left.A_{2} \frac{\partial F}{\partial x}+B_{2} \frac{\partial F}{\partial y}+\varphi_{2}^{\prime} x, y\right)}=\frac{d y}{d x} \text { oder }
$$

$A_{2} \frac{\partial F}{\partial x} \sin \varphi+B_{2} \frac{\partial F}{\partial y} \sin \varphi-A_{1} \frac{\partial F}{\partial x} \cos \varphi-B_{1} \frac{\partial F}{\partial y} \cos \gamma^{\prime}=\varphi_{1}(x, y) \cos \varphi-\varphi_{2}(x, y) \sin \varphi$.

Es ist klar, daß der Ausdruck auf der linken Seite dann und nur dann mit dem Differentialquotienten in Richtung der Berandung (d.h.: $\frac{\partial}{\partial)_{8}}$ ) iibereinstimmt beziehungew. diesem proportional ist, wenn $A_{2}=B_{1}=0$ and $\left.A_{1}=-B_{3} \neq 0^{1}\right)$. Setzen wir die Konstante $A_{1}=-B_{2}=-1-$ was, wie man sofort einsieht, keine wesentliche Einschränkung mehr bedentet - und $A_{2}=B_{1}=0$, so nimmt unser Gleichungssystem (F) folgende Form an:

1) Würde man hingegen $A_{1}=B_{2}=0$ und $A_{2}=B_{1} \neq 0$ setzen, so flele die linke Seite proportional $\frac{\partial F}{\partial}$ aus, also wäre der derart spezialisierte Ansatz $(F)$ eine nur unwesentliche und von unserem standpunkte ans zur Lösung des Problems niebts beitragente Verallgemeinerung des Ansatzes ( $\left.\Psi^{\prime}\right)$. 


$$
\left.\begin{array}{c}
\tau_{y z}=-\frac{\partial F}{\partial x}+\phi_{1}(x, y) \\
\tau_{x x}=\frac{\partial F}{\partial y}+\varphi_{2}(x, y)
\end{array}\right\}
$$

die Randbedingung gestaltet sich dabei wie folgt:

$$
\frac{\partial F}{\partial y} \sin \varphi+\frac{\partial F}{\partial x} \cos \varphi \equiv \frac{\partial F}{\partial s}=\varphi_{1}(x, y) \cos \varphi-\varphi_{2}(x y) \sin \varphi
$$

und demzufolge (abgesehen von einer für unser Problem - im Falle einfach zusammenhängender Querschnitte - bedeutungslosen Konstanten):

$$
F(s)=\int_{0}^{s}\left[\varphi_{1}(x, y) \cos \varphi-\varphi_{2}(x y) \sin \varphi\right] d s
$$

wenn $F(s)$ den Wert der Funktion $F$ an jenem Punkt der Berandung bedeutet, welcher von einem auf diesem angenommenen festen Punkt $A$ die Bogenentfernung $s$ aufweist. Die Funktion $F(s)$, d. h. der Verlauf von $F(x, y)$ längs des Randes, läßt sich duroh graphische Integration ermitteln, sobald die Funktionen $\varphi_{1}$ und $\varphi_{2}$ gewählt sind und der Rand bekannt ist. Denken wị uns die so gefundenen Werte von $F$ über den Randpunkten tatsächlich aufgetragen, so erhalten wir die Raumkurve, längs der die Fläohe $F$ die Mantelfläche schneidet.

Sehen wir nun zu, welcher partiellen Differentialgleichung die Funktion $F$ genügt.

Wenden wir auf $F$ die Operation $A_{x y}{ }^{2}=\frac{\partial^{2}}{\partial x^{2}}+\frac{\partial^{2}}{\partial y^{2}}$ an:

$$
\begin{aligned}
d_{x y}{ }^{2} F & \equiv \frac{\partial^{2} F}{\partial x^{2}}+\frac{\partial^{2} F^{2}}{\partial y^{2}}=-\frac{\partial \tau_{y z}}{\partial x}+\frac{\partial \varphi_{1}}{\partial x}+\frac{\partial \tau_{x z}}{\partial y}-\frac{\partial \varphi_{2}}{\partial y} \\
& =-C \frac{\partial^{2} \Psi}{\partial x \partial y}+C(2 m-1) x+\frac{\partial \varphi_{1}}{\partial x}+C \frac{\partial^{2} \Psi}{\partial x} \frac{\partial y}{\partial x}-C(2 m+1) x-\frac{\partial \varphi_{2}}{\partial y} \\
& =-2 C x+\frac{\partial \varphi_{1}}{\partial x}-\frac{\partial \varphi_{2}}{\partial y}\left(l^{\prime}\right) .
\end{aligned}
$$

Wenn wir also ein für allemal ein Funktionenpaar $\varphi_{1}, \varphi_{2}$ annohmen, so läßt sich aus der partiellen Differentialgleichung $\left(J^{\prime}\right)$ und der Randbedingung ( $d^{\prime}$ ) für jede beliebige Berandung jene Funktion $F$ bestimmen, aus der, vermittels des Gleiohungssystems (F') die Schubspannungskomponenten sich ableiten lassen.

Die Funktionen $\varphi_{1}$ und $\varphi_{2}$ dürfen aber nicht ganz beliebig gewählt werden, wenn wir die Funktion $F$ samt ihren ersten und zweiten partiellen Ableitungen kontinuierlich haben wollen. Nach einem wohlbekannten Satze ist die notwendige Bedingung dieser Kontinuitätseigenschaften: $\frac{\partial}{\partial x}\left(\frac{\partial F}{\partial y}\right)=\frac{\partial}{\partial y}\left(\frac{\partial F}{\partial x}\right)$. Das verlangt aber einen gewissen Zusammenhang zwischen $\varphi_{1}$ and $\varphi_{2}$. Nämlich

also

$$
\begin{aligned}
& \frac{\partial}{\partial x}\left(\frac{\partial F}{\partial y}\right)=\frac{\partial \tau_{x x}}{\partial x}-\frac{\partial \varphi_{2}}{\partial x}=\left(C \frac{\partial^{2} \Psi}{\partial x^{2}}-C(2 m+1) y\right)-\frac{\partial \varphi_{2}}{\partial x} \\
= & \frac{\partial}{\partial y}\left(\frac{\partial F}{\partial x}\right)=-\frac{\partial \tau_{y x}}{\partial y}+\frac{\partial \varphi_{1}}{\partial y}=\left(-C \frac{\partial^{2} \Psi}{\partial y^{2}}+C y\right)+\frac{\partial \varphi_{1}}{\partial y},
\end{aligned}
$$

$$
O=C \frac{\partial^{2} \Psi}{\partial x^{2}}+C \frac{\partial^{2} \Psi}{\partial y^{2}}-C(2 m+1) y-C y-\frac{\partial \varphi_{2}}{\partial x}-\frac{\partial \varphi_{1}}{\partial y},
$$

woraus, unter Anwendnng der Gleichung $(d)$, folgt, daß

$$
2 C(m+1) \boldsymbol{y}+\frac{\partial \varphi_{2}}{\partial x}+\frac{\partial \varphi_{1}}{\partial y}=0
$$

Die Fanktionen $\varphi_{1}$ und $\varphi_{2}$ dürfen nur so gewählt werden, daB die Relation (K) erfüllt ist. Um nun unsere Ergebnisse vom Standpunkte der praktisohen Lösung möghiohst günstig zu gestalten, müssen wir über $\phi_{1}$ und $q_{2}$ - natïrlioh unter Beriucksichtigung von (K) - zweckmäßig disponieren. Nachdem in Folge von (K) $\varphi_{1}$ und $\varphi_{2}$ nicht beide lineare Funktionen von $x$ und $y$ sein könuen, erscheint es mir zweckmäßig, beide Funktionen als homogene quadratische Funktionen der genannten Veränderliohen zu wählen, also:

$$
\left.\begin{array}{l}
\varphi_{1}(x, y)=a_{1} x^{2}+b_{1} x y+c_{1} y^{2} \\
\varphi_{2}(x, y)=a_{2} x^{2}+b_{2} x y+c_{2} y^{2}
\end{array}\right\}
$$


dann erhält (K) folgende spezielle Form:

$$
2 C(m+1) y+2 a_{2} x+b_{2} y+b_{1} x+2 e_{1} y=0
$$

für alle reellen Wertpaare $x, y$; daher folgt:

$$
\left.\begin{array}{c}
2 C(m+1)+b_{2}+2 c_{1}=0 \\
2 a_{2}+b_{1}=0
\end{array}\right\}
$$

Im Folgenden werden wir - natürlich unter Berücksichtigung von $\left(K^{*}\right)$ - zwei - nach verschiedenen Gesichtspunkten zweckmäßige - Verfügungen iiber die homogenquadratischen Funktionen $\varphi_{1}$ und $\varphi_{2}$ vornehmen. Im Falle der ersten Wahl entspricht jeder Berandung eine ganz bestimmte Funktion $F$, die wir zur Unterscheidung $F_{I}$ nennen wollen, bei der zweiten Wahl eine andere ganz bestimmte Funktion, die $F_{I T}$ genannt werden soll.

2. Erste Verfügung über $\varphi_{1}$ und $\varphi_{2}$. Das einfachste Paar solcher homogen-quadratischer Funktionen, deren Koeffizienten dem Gleichungssystem ( $\left.K^{*}\right)$ genügen, ist $\Phi_{1}=0 ; \Phi_{2}=-2 C(m+1) x y$. In diesem Falle ist:

$$
\frac{\partial \varphi_{1}}{\partial x}=0 ; \frac{\partial \varphi_{2}}{\partial y}=-2 C(m+1) x
$$

Daher erhält das Hauptergebnis in 1 (siehe die Gleichnngen $\left(F^{\prime}\right),\left(d^{\prime}\right),\left(\Delta^{\prime}\right)$ ) folgende speziellere Fassung: die Fanktion $F_{I}$, aus welcher die Schubspannungskomponenten vermittelst der Gleichungen

$$
\left.\begin{array}{rl}
\tau_{y z} & =-\frac{\partial F_{I}}{\partial x} \\
\tau_{x z} & =\frac{\partial F_{I}}{\partial y}-2 C(m+1) x y
\end{array}\right\}
$$

folgen, genügt in jedem inneren Punkte der Querschnittsfläche der partiellen Differentialgleichang

$$
\lrcorner_{x y}^{2} F_{I} \equiv \frac{\partial^{2} F_{I}}{\partial x^{2}}+\frac{\partial^{2} F_{I}}{\partial y^{2}}=2 C m x \text {. . . . . . . . . . }
$$

und nimmt in jedem Randpunkte des Querschnittes - von einer additiven Konstanten abgesehen - den Wert

$$
F_{2}(s)=\int_{0}^{s} \lambda_{r}(s) d s=\int_{0}^{s} 2 C(m+1) x y \text { sin } \varphi d s
$$

an. Die Kurve $F_{I}(s)$, in welcher die Fläche $F_{I}$ die Mantelfläche des Prismas schneidet, läßt sich danaoh bei gewissen Quersohnitten rechnerisch ermitteln, in allen Făllen, insbesondere bei nur zeichnerisch gegebenen, nach dem bekannten Verfahren graphischer Quadratur zeichnerisoh bestimmen.

(Im Falle einer Quersohnittsberandung, die aus lauter zu den beiden Koordinatenachsen $x$ und $y$ parallelen Geradenstücken besteht, - wie z. B. bei dem Reokteck und, abgeseben von den Abrundungen and Abstumpfungen, anch bei den technisch wichtigen T, I und Kreuzquerschnitten, - besteht die Raumkurve $F_{I}(s)$ aus lauter zur $x$-Achse parallelen Geradenstïcken and ans Stücken von Parabelbögen zweiter Ordnung, wis man aus der Formel $\left(d_{l}\right)$ sofort sieht.)

Durch obige Formulierung unserer Ergebnisse wird es nun sofort klar, dab zur Bestimmung der Funktion $F_{I}$, welche unmittelbar durch die Gleichungen $\left(\mathrm{d}_{t}\right)$ und $\left(\Delta_{I}\right)$ gegeben ist, genau dieselbe numerisch-graphische Methode anwendbar ist, welche $R$ unge zur Bestimmung der die Prandtlsche Lösung des Torsionsproblems liefernde Funktion gesohaffen hat. Man sieht sogar, daB man bei jedem beliebigen Querschnitt durch die Lösung des Torsionsproblems in seiner Prandtlschen Fassung und des Biegungsproblems in unserer obigen Fassung auf zwei solche lineare Gleichungssysteme geführt wird, welche miteinander ihrer straktur ${ }^{1}$ ) nach vollständig übereinstimmen.

1) Die Strukture eines Gleichungssystems ist im wesentlichen eine graphostatische Begriffsbildung. Zwei Systeme ron linearen Gleichungen werden dann als Gleichungssysteme uhereinstimmender Straktur bezeichnet, wenn die Anzahl der Unbekannten abereinstimmt, und wenn die Gleichungen der beiden Systeme sieh in eine ein-eindeutige Beziebung bringen Jassen derart, dab in zwei korrespondierenden Gleichnngen die namlichen Unbekannten mit einem von 0 verschiedenen Koeffizienten behaftet sind. Leisten wir bei dem Aufstellen der Gleichungssysteme auf die oventuellen Symmetrie-Vereinfachungen Verzicht, so ist in diesem Sinne die Gleichheit der Struktur der beiden im Texte erwhhnten Gleichungssysteme unmittelbar einleuchtend. 
3. Andere Verfügung über $q_{1}$ und $q_{2}$. $\dot{Z u}$ einer andern Lösungsmethode gelangen wir. indem wir setzen: $\varphi_{1}=C\left[x^{2}-(m+1) y^{2}\right] ; \gamma_{2}=0$, also $a_{1}=C, b_{1}=0, c_{1}=$ $-C(m+1) ; \alpha_{2}=b_{2}=c_{2}=0$; womit die Bedingung $\left(K^{*}\right)$ befriedigt ist. In diesem Falle ist:

$$
\frac{\partial \varphi_{1}}{i x}=2 C x ; \quad \frac{\partial \varphi_{2}}{\partial y}=0
$$

and man sieht sofort, daB in diesem Falle das entsprechende $F$, das zur Unterscheidung $F_{I I}$ genannt werden soll, der Laplaceschen Differentialgleichung zu genügen hat.

Das Hauptergebnis in 1 (siehe die Gleichungen $\left.\left(\mathrm{F}^{\prime}\right),\left(\mathrm{d}^{\prime}\right),\left(f^{\prime}\right)\right)$ erhält also hier folgende spezielle Fassung: die Funktion $F_{I}$, aus welcher die Schubspannungskomponenten vermittelst der Gleichungen:

$$
\begin{aligned}
& \tau_{y z}=-\frac{\partial F_{I I}}{\partial x}+C\left(x^{2}-(m+1) y^{2}\right) \\
& \tau_{x z}=\frac{\partial F_{I I}}{\partial y}
\end{aligned}
$$

folgen, genügt in jedem inneren Pankte der Querschnittsfläche der La placeschen Differentialgleichuug

$$
J_{x y}^{2} F_{I I}=\frac{\gamma^{2} F_{I I}}{\partial x^{2}}+\frac{\iota^{2} F_{I I}}{i y^{2}}=0 \quad \cdot \quad \cdot \quad \cdot \quad \cdot \quad \cdot \quad \cdot \quad \cdot\left(\Delta_{I I}\right)
$$

und nimmt in jedem Randpunkte des Querschnitts - von einer additiven Konstanten abgesehen - den Wert

$$
F_{I I}(s)=\int_{0}^{s} \lambda_{I I}(s) d s=\int_{0}^{s} C\left(x^{2}-(m+1) y^{2}\right) \cos \varphi d s .
$$

an. Die Kurve $F_{I I}(s)$, in welcher die Fläche $F_{I I}$ die Mantelfläche des Prismas schneidet, läbt sich ebenso wie $F_{I}(s)$ durch einfache Quadratur bestimmen. (Im Falle einer aus lauter zu den beiden Koordinatenachsen $x$ und $y$ parallelen Geradenstïcken bestehenden Querschnittsberandung besteht die Kurve $F_{I I}(s)$ aus lauter zur $y$.Achse parallelen Geradenstücken und aus Stücken von Parabeln III. Ordnung, wie aus $G l .\left(d_{I I}\right)$ sofort ersichtlich ist.)

Die Grenzbedingung ist hier etwas weniger einfach als bei der in 2. erörterten Methode mit der Funktion $F_{X}^{\prime}$; daB wir trotzdem auch $F_{I Y}$ einführten, ist dadurch begründet, daB $F_{I I}$ der Laplaceschen Differentialgleichung genügt. Dieser Umstand ermöglicht uns eine experimentelle Darstellung der Fläche $F_{1 I}$.

Stellen wir eine ganz beliebig geschlossene Raumkurve durch einen dünnen, in sich zurückkehrenden Draht dar und tauchen denselben in eine Seifenlösung, so spannt sich auf den Draht eine Flüssigkeitsmembran. welche - von der geringfügigen störenden Wirkung der Schwerkraft abgesehen - die Form einer sogenannten Minimalfläche besitzt, also einer durch die Gleichung

$$
\frac{1}{\varrho_{1}}+\frac{1}{\varrho_{2}}=0
$$

gekennzeichneten Fläche ${ }^{1}$ ) (wo $\varrho_{1}$ und $\varrho_{2}$ die Hauptkrümmungsradien bedeuten).

Wenn wir für diese Minimalfläche die Gleichung $f=f(x, y)$ einführen, so läbt sich - (wie es in der Differentialgeometrie gezeigt wird) - GI. (m) in folgender Form anschreiben:

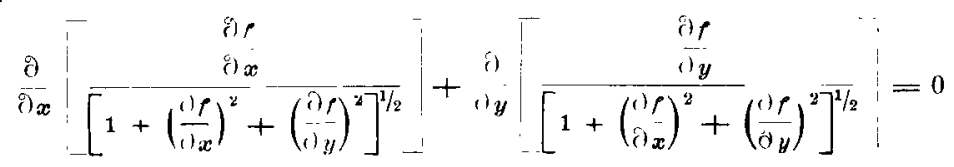

oder nach einiger Umformung

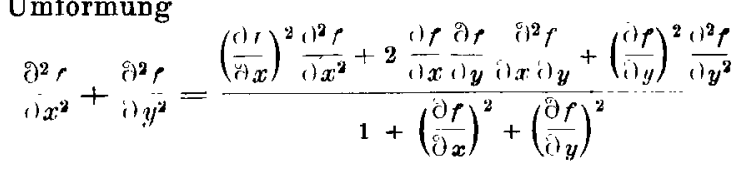

Aus dieser letzteren Form folgt, daß, sobald die Minimalfläche $f$ so flach geneigt ist, daß die Quadrate und das Produkt ans $\frac{\partial f}{\partial x}$ und $\frac{\partial f}{\partial y}$ der Einheit gegenüber vernach-

1) siehe z. B. das Kompendium der theor. Phrsik ron Woldemar Voigt, Bd. I, S. 248. 
lässigt werden dïrfen, sie mit zureichender Genanigkeit der Laplaceschen Differentialgleichung genügt.

Stellen wir daher vermittelst des oben angeführten Kapillarexperimentes jene Minimalfläche $f$ her, welche die dem vorgelegten Stabquerschnitt enteprechende Raumkurve $F_{I T}(s)$ enthält, und sehen wir, daß die derart gewonnene Fläche eine geringe Neignng zur $x, y$-Ebene aufweist, so wissen wir, daß diese Minimalfläche annähernd die Laplacesche Differentialgleichung befriedigt; daher stimmt Fläche $f$ mit $F_{I I}$ genan in der Grenzbedingung und näherungsweise in der Differentialgleichung überein, so daß die Fläche $f$ eine Näherungslösung unseres Problems liefert.

Hat hingegen die zur Ranmkurve $F_{I I}(s)$ gehörende Minimalfläche $f$ irgendwo eine so große Neigung, daß Quadrate und Produkt von $\frac{\partial f}{\partial x}$ und $\frac{\partial f}{\partial y}$ nicht vernachlässigt werden dürfen, so müssen wir für unser Biegungsproblem eine andere approximative Lösungsmethode suchen. Als eine solche bietet sich auch hier Runges numerisch-graphisches Verfahren dar, welches auch hier - wie sofort einzusehen - auf ein Gleichungssystem gleicher Struktur führt, wie das Torsionsproblem in seiner Prandtlschen Fassung und das Biegungsproblem in seiner in 2. von uns entwickelten Formulieruog.

Eine weitere Nuherungsmethode orhält man, wenn man diese numerisch-graphische Methode mit der obigen experimentellen Methode derart kombiniert, daß man die Gitterpunktwerte der experimentell ermittelten Funktion $f$ als erste Annäherungswerte für die Lösung unseres Rungeschen Gleichungssystems betrachtet und von da aus mit dem bekannten Iterationsverfahren bis zum vorgeschriebenen Genauigkeitsgrade fortschreitet.

4. Anwendung auf den Grashofschen Querschnitt. Weiterer Ausblick. Zum Schlu $B$ will ich eine Bemerkung über die geschlossene Raumkurve $F_{I I}(s)$ machen, diese Bemerkung auf eine spezielle Querschnittsform, den sogenannten Grashofschen Querschnitt, anwenden und für diesen das Spannungsproblem lösen.

Die Kurve $F_{I I}(s)$ ist bekanntlich eine ebene Kurve, falls die Determinante

$$
\left.\Delta(s)=\left|\begin{array}{ccc}
\frac{d x}{d s} & d y & d F_{I I} \\
\frac{d s}{d s} \\
\frac{d^{3} x}{d s^{2}} & d^{2} y & d^{2} F^{2} I \\
\frac{d s^{2}}{d s^{2} x} & \frac{d^{3} y}{d s^{3}} & \frac{d^{3} F_{I}}{d s^{3}}
\end{array}\right|=\mid \begin{array}{ccc}
\frac{d x}{d s} & \frac{d y}{d s} & \left(\frac{d x}{d s}\left(x^{2}-(m+1) y^{2}\right)\right. \\
\frac{d^{3} x}{d s^{2}} & \frac{d^{3} y}{d s^{2}} & \left(: \frac{d}{d s}\left[\frac{d x}{d s}\left(x^{2}-(m+1) y^{2}\right)\right]\right. \\
\frac{d^{3} x}{d s^{3}} & \frac{d^{3} y}{d s^{3}} & C \frac{d^{2}}{d s^{2}}\left[\frac{d x}{d s}\left(x^{2}-(m+1) y^{2}\right)\right]
\end{array}\right]
$$

für jeden beliebigen Wert des Parameters $s$ gleich 0 ist.

Setzen wir also die Determinante $\Delta(s)$ gleich 0 , nehmen wir ferner zur hierdurch erhaltenen Gleichung die Identitat $\left(\frac{d x}{d s}\right)^{2}+\left(\frac{d y}{d s}\right)^{2}=1$ hinzu, und lösen wir dieses System von Differentialgleiohungen auf, so erbalten wir eine mehrdimensionale Schar solcher Querschnittskurven, für welche die Karve $F_{I I}(s)$ eine ebene Kurve und demzafolge wie man unter Heranziehung der Gleichung $\left(d_{I I}\right)$ unmittelbar folgert - die Flüche $F_{I I}$ eine Ebene ist; für jede solche Querschnittsbegrenzung ist also das Spannungsproblem elementar lösbar. Von dieser mehrdimensionalen Kurvenschar greifen wir eine eindimensionale Hyperbelschar mit der Gleichung

$$
x^{2}-(m+1) y^{2}=-k^{2}
$$

- wo $k$ der Parameter der Kurvenschar - heraus; man sieht sofort, dab für jede Hyperbel dieser Schar die dritte Kolonne der Determinante $J(s)$ mit der ersten übereinstimmt und daher die Bedingung $\Delta(s)=0$ in der Tat befriedigt ist. Weiter ist es leicht einzusehen, daß auch dem hieraus gebildeten sogenannten Grashof schen Querschnitt ) - (oben und unten begrenzt durch die beiden Aeste einer der Hyperbeln (h), an den beiden anderen Seiten durch zwei zur $y$-Achse parallel und symmetrisch gelegenen Geraden) - die Eigenschaft zukommt, daB die ihm entsprechende Kurve $F_{I I}(s)$ einer einzigen Ebene angehört. Diese Ebene, welche gleichzeitig die Fläche $F_{I I}$ liefert, wird, wie leicht nachweisbar, durch die Gleichung

$$
F_{I I}=C k^{2} x
$$

') siehe z. B Lorenz, a. a. O., S. 442. 
dargestellt; sie enthält also die $y$-Achse und gibt die Lösung des Biegungsproblems für den Grash of schen Querschnitt, nämlich nach $\left(\mathrm{F}_{I I}\right)$ :

$$
\begin{aligned}
& \tau_{y z}=-\frac{\partial F_{n}}{\partial x}+C\left(x^{2}-(m+1) y^{2}\right)=-C k^{2}+C\left(x^{2}-(m+1) y^{2}\right) \\
& =C\left(x^{2}-(m+1) y^{2}-k^{2}\right) \\
& \tau_{x z}=\frac{\partial F_{I I}}{\partial y}=0 .
\end{aligned}
$$

Wir sind damit auf ganz anderem Wege zu dem bekannten Grashofschen Ergebnis gelangt.

Die in denselben Problemkreis fallenden weiteren Erörterungen werden nun zunächst zwei Ziele zu verfolgen haben:

1) Die tatsächliche Lösung des Biegungsproblems auf Grund der Ergebnisse von 2 and 3 mit Hilfe unserer Funktionen $F_{1}$ und $F_{I I}$ fär technisch wichtige und bisher der exakten Lösung unzugängliche Querschnitte, - in erster Linie für das $T$, das $I$, das kreuzförmige und das Zorés-Profil; ferner für theoretisch interessante Querschnitte, so in erster Linie das Rechteck und das diagonal gestelite Quadrat. Bei diesen letzteren wird sich ein Vergleich der derart zu gewinnenden Ergebnisse mit den Ergebnissen der willkürlichen "Naherungsmethode" (siehe Einleitung und Fußn. 5, S. 90) darbieten.

2) Die Erweiterung des Rungeschen graphisch-numerischen Lösungsverfahrens des Torsionsproblems ${ }^{1}$ ) auf $z$ weifach and mehrfach zusammenhängende Querschnitte; Anwendung hiervon auf den Stab mit rahmenförmigem Querschnitt (rechteokiges Hohlprisma) und die analoge Untersuchung in bezng auf das Biegungsproblem, ebenfalls unter Anwendung auf das rechteckige Hohlprisma.

\title{
über eine Eigenschaft der ballistischen Kurve und ihre Anwendung auf die Integration der Bewegungsgleichungen.
}

\author{
Von K. POPOFF in Sofia.
}

$\mathrm{H}$ enri Poincaré hat in zwei seiner Arbeiten, nämlich in den "Méthodes nouvelles de la Mécanique céleste " und in den "Leçons de Mécanique céleste", wertvolle Methoden zur Integration der Differentialgleichungen der Himmelsmechanik angegeben. Im folgenden soll versucht werden, diese Methoden auch auf die Differentialgleichungen der Ballistik anzuwenden. Wrhrend aber alle in der Himmelsmechanik auftretenden Krafte sich aus einem Potential ableiten lassen, gibt in der Ballistik die Rei . bung dem Problem eine gänzlich veränderte Gestalt. Sieht man ferner die Reibungskräfte als die *störenden Kräfte« im Sinne der Himmelsmechanik an, so sind diese hier unverhältnismß̈Big viel größer als in der Himmelsmechanik.

Für das Problem der Himmelsmechanịk findet nun Poincaré folgendermaßen eine allgemeine Lösung. Fr ermittelt zunächst Hilfslösungen (solutions intermédiaires), die Eigenschaften haben, die einem Spezialfall des Problems entsprechen, aber bis zu einem gewissen Grade auch im allgemeinen Falle auf́reten müssen. Von diesen, übrigens bei ihm periodischen oder asymptotischen, Hilfslösungen geht er dann mit Hilfe der sogen. Störungsgleichungen (équations aux variations) zur Lösung des allgemeinen Problems über.

Es kann der Fall eintreten, daß die Anfangsbedingungen der gesuchten Bewegung nur wenig von denen abweichen, welche einer (periodischen) Hilfslösung entsprechen. In diesem Falle kann man diese Lösung als erste Näherung der wahren Lösung be-

1) Runges numerisch-graphisches Verfahren ließe sich ganz unverỉndert auf mehrfach zusammenhängende Quersehnitte uhertragen, falls man im vorlinein fur jede innere geschlossene Randkurve des mehrfach zusammenhüngenden Querschnitte den ilı eigenen konstanten wert der Funktion $u(x, y$, - also bei einem n-fach zusammenhäneenden Querschnitte $n-1$ Kunatanie - kennen wïrie; dies ist aber zunächst nfeht der Fall Die Ueberwlodung der hierin liegenden Schwlerigkeit und damit die Verallgemeinerung der Rungeschen Lösung des Torsonsproblems auf beliebig-rielfach zusammenhängende Querschnitte blldet den Gegenstavd elner in Vorbereitung befindlichen Abhandlung des Verfassers. 